\title{
Targeting Cellular DNA Damage Responses in Cancer: An In Vitro-Calibrated Agent-Based Model Simulating Monolayer and Spheroid Treatment Responses to ATR-Inhibiting Drugs
}

\author{
Sara Hamis $^{1,2}$ (D) James Yates $^{3} \cdot$ Mark A. J. Chaplain $^{1} \cdot$ Gibin G. Powathil $^{2}$ \\ Received: 8 May 2020 / Accepted: 10 August 2021 \\ (c) The Author(s) 2021
}

\begin{abstract}
We combine a systems pharmacology approach with an agent-based modelling approach to simulate LoVo cells subjected to AZD6738, an ATR (ataxia-telangiectasiamutated and rad3-related kinase) inhibiting anti-cancer drug that can hinder tumour proliferation by targeting cellular DNA damage responses. The agent-based model used in this study is governed by a set of empirically observable rules. By adjusting only the rules when moving between monolayer and multi-cellular tumour spheroid simulations, whilst keeping the fundamental mathematical model and parameters intact, the agent-based model is first parameterised by monolayer in vitro data and is thereafter used to simulate treatment responses in in vitro tumour spheroids subjected to dynamic drug delivery. Spheroid simulations are subsequently compared to in vivo data from xenografts in mice. The spheroid simulations are able to capture the dynamics of in vivo tumour growth and regression for approximately 8 days post-tumour injection. Translating quantitative information between in vitro and in vivo research remains a scientifically and financially challenging step in preclinical drug development processes. However, well-developed in silico tools can be used to facilitate this in vitro to in vivo translation, and in this article, we exemplify how data-driven, agent-based models can be used to bridge the gap between in vitro and in vivo research. We further highlight how agent-based models, that are currently underutilised in pharmaceutical contexts, can be used in preclinical drug development.
\end{abstract}

SH was supported by the Medical Research Council [Grant Code MR/R017506/1] and Swansea University PhD Research Studentship.

$凶 \quad$ Sara Hamis

sjh37@st-andrews.ac.uk

1 School of Mathematics and Statistics, University of St Andrews, St Andrews, UK

2 Department of Mathematics, Swansea University, Swansea, UK

3 Oncology R\&D, AstraZeneca, Cambridge, UK 
Keywords DNA damage response inhibition - Agent-based model $\cdot$ Mathematical oncology · AZD6738

\section{Introduction}

\subsection{Bridging In Vitro and In Vivo Research}

Mathematical models, and their corresponding in silico tools, can be used to simulate both in vitro and in vivo scenarios that involve cancer cell populations, or tumours, and their responses to anti-cancer treatments (Rockne et al. 2019; Bruno et al. 2020; Stephanou et al. 2018; Brady-Nicholls et al. 2020; Scott et al. 2020). However, cancer cells in an in vitro cell culture experience a microenvironment that is significantly different from the microenvironment experienced by cancer cells in a solid tumour in vivo. As these microenvironments influence cell proliferation and the delivery of oxygen, drug and nutrient molecules to cells, it follows that the dynamics of a cancer cell population in vitro differs from the dynamics of a solid tumour in vivo. Consequently, translating data obtained by in vitro experiments into quantitative information that can guide or predict in vivo experiments remains a challenging, but important, step in drug development processes. As an intermediate step between monolayer cell cultures and in vivo tumours, multi-cellular tumour spheroids (in this study referred to as spheroids) provide in vitro models that are able to capture certain key-features of in vivo tumours such as intratumoural heterogeneity resulting from nutrient-gradients and resource-limited tumour growth (Nunes et al. 2019).

Agent-based models (ABMs) are used in many applications in mathematical biology but are underutilised in the context of pharmaceutical drug development (Cosgrove et al. 2015). An ABM consists of multiple, distinct agents that may interact with each other and their microenvironment. There exist different types of ABMs. For example, agents can be deformable or of fix size, and agent movements and neighbourhoodinteractions can be constrained by an underlying lattice geometry (on-lattice models) or not (off-lattice models). Combining ABMs with hybrid modelling techniques allows for the integration of discrete and continuous variables describing tumour dynamics on multiple scales. A thorough review on various types of hybrid ABMs used to simulate tumour growth is provided by Rejniak and Anderson (2011). Furthermore, a number of open-source in silico tools, such as Chaste (Mirams et al. 2013), CompuCell3D (Swat et al. 2012) and PhysiCell (Ghaffarizadeh et al. 2018), are freely available to facilitate the implementation of ABMs.

In this study, we introduce a novel modelling approach that uses an agent-based mathematical model to bridge the gap between in vitro monolayer and spheroid research as a step towards bridging the gap between in vitro and in vivo research, as conceptually illustrated in Fig. 1. For a broader scope discussion on how to develop, calibrate and validate mathematical models that can predict novel anti-cancer therapies, we refer the reader to a recent article by Brady and Enderling (2019). In the $\mathrm{ABM}$ at the core of this modelling approach, an agent consists of one cancer cell or a group of cancer cells, where the behaviour and fate of each agent are governed by a set of empirically observable and well-established modelling rules that incorporate 


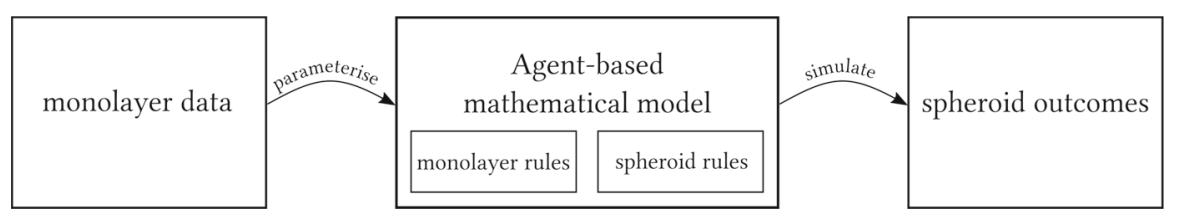

Fig. 1 A schematic of the mathematical modelling approach used in this study. An agent-based mathematical model, that distinguishes between in vitro monolayer and spheroid modelling rules, is formulated. The mathematical model is first parameterised by in vitro monolayer data and is thereafter used to simulate spheroid dynamics

both intracellular and microenvironmental dynamic variables, as described throughout Sect. 2. To account for differences between monolayer and spheroid scenarios, the modelling rules are adjusted when moving between monolayer and spheroid simulations. By only adjusting the rules, whilst keeping the fundamental mathematical model and parameters intact, when moving between monolayer and spheroid simulations, the mathematical framework can first be parameterised by monolayer data and thereafter be used to simulate spheroid treatment responses. To exemplify this modelling approach, we here simulate LoVo (human colon carcinoma) cells subjected to the anti-cancer drug AZD6738. The ABM is first calibrated by monolayer in vitro data and is thereafter used to simulate in vitro spheroids subjected to dynamic drug delivery. Spheroid simulations are subsequently compared to xenograft in vivo data. The in vitro and in vivo data used in this study are gathered from previous work by Checkley et al. (2015). The ABM used in this study is an extension of a model introduced by Powathil et al. (2012b).

\subsection{DNA Damage Response Inhibiting Drugs}

The deoxyribonucleic acid (DNA) in human cells is perpetually exposed to, potentially harmful, influences that can be derived from both exogenous and endogenous sources and events (Minchom et al. 2018; Sundar et al. 2017). Exogenous sources include ultraviolet radiation, ionising radiation and chemotherapeutic drugs, whilst erroneous DNA replication is an example of an endogenous event yielding DNA damage (Minchom et al. 2018). Regardless of the source, a multitude of intracellular events are triggered when the DNA in a cell becomes damaged. Cells may, for example, respond to DNA damage by activating DNA repair mechanisms, cell cycle arrest or, in cases of severe DNA damage, apoptosis (Carrassa and Damia 2017). Such cellular responses to DNA damage are mainly governed by the DNA damage response (DDR), which comprises a complex network of signalling pathways (Carrassa and Damia 2017). The DDR has many functionalities and, amongst other things, it monitors DNA integrity and repairs DNA damage in order to maintain genomic stability in cells. The DDR also governs DNA replication, cell cycle progression and apoptosis (Minchom et al. 2018; Nam et al. 2019). When DNA repair in a cell is needed, the DDR activates relevant effector proteins (Minchom et al. 2018). Included in the group of DDR-associated effector proteins are approximately 450 proteins (Nam et al. 2019), out of which the two main regulators for cell cycle checkpoints are ataxia-telangiectasia-mutated kinase (ATM) and ataxia- 
telangiectasia-mutated and rad3-related kinase (ATR) (Sundar et al. 2017). ATM and ATR belong to the enzyme family phosphatidyilinositol-3-OH-kinases (PI3K), and they both play central roles when cells respond to DNA damage (Carrassa and Damia 2017). In this work, we study the effects of an anti-cancer drug, namely AZD6738, that works by inhibiting ATR activity.

DNA lesions in form of single-strand breaks are a common result of replication stress, and the repair of single-strand DNA breaks is mainly attributed to ATR activity. A drug that inhibits ATR activity consequently inhibits the repair of single-strand DNA breaks post-replication stress. Cancer cells are associated with high replication stress and consequently ATR inhibitors have, during the last decade, been explored as anti-cancer agents (Minchom et al. 2018; Carrassa and Damia 2017; Mei et al. 2019). With the premise that inhibiting DNA damage responses should increase the effect of some other main therapy, DDR inhibitors have been explored as both radiotherapy and chemotherapy treatment intensifiers (Carrassa and Damia 2017; Mei et al. 2019). Two well-studied ATR inhibitors are AZD6738 and VX-970. AZD6738 is an oral ATR inhibitor, and its anti-tumour potential has been demonstrated in preclinical vitro and in vivo xenograft studies for various ATM-deficient cell lines, including ATMdeficient lung cancer, chronic lymphocytic leukaemia and metastatic adenocarcinoma of the colon (Checkley et al. 2015; Sundar et al. 2017; Foote et al. 2018). Combination treatments that combine AZD6738 with either radiotherapy or chemotherapy have produced synergistic results in preclinical settings (Sundar et al. 2017), and AZD6738 is currently being evaluated in clinical phase I/II trials (Minchom et al. 2018; Mei et al. 2019). VX-970 is an intravenous ATR inhibitor (Tu et al. 2018) that has demonstrated tumour controlling effects in a phase I clinical trial, both as a monotherapy and in combination with the chemotherapy drug carboplatin (Minchom et al. 2018). A summarising table of clinical trials involving ATR-inhibitors can be found in an article by Mei et al. (2019).

\section{Model and Method}

We use an ABM approach to model monolayer populations of cancer cells and multicellular tumour spheroids that evolve in time and space. The model describes the behaviour of cancer cells using a set of modelling rules. In order to account for differences between monolayer and spheroid scenarios, these rules are adjusted when moving between monolayer and spheroid simulations, as described throughout Sect. 2. Taking a minimal parameter approach, we aim to use as few rules and parameters as possible to capture the nature of the regarded systems. We here chose to include model rules and parameters that pertain to the cells' doubling time and cell cycle (Sect. 2.2), cell proliferation on the lattice (Sect. 2.3), the distribution of oxygen and drugs across the lattice (Sects. 2.4 and 2.5, respectively) and cellular responses to local oxygen and drug concentrations (Sects. 2.4 and 2.6, respectively). In this work, details concerning nutrient distribution and its effect on tumour growth are not included. Instead, under a simplifying modelling assumption, the diffusion of oxygen forms a surrogate for the distribution of nutrients. Differences between monolayer and spheroid simulation 
modelling rules are pictorially summarised in Sect. 2.8, and monolayer-calibrated model parameters are listed in Sect. 2.7.

The in vitro and in vivo data used in this study are gathered from previous work by Checkley et al. (2015). In the regarded in vitro experiments, populations of LoVo cells were plated and subjected to AZD6738, where population sizes of up to roughly 4000 cells were reported (Checkley et al. 2015). In the in vivo experiments, LoVo cells were subcutaneously injected in flanks of female Swiss nude mice in order to produce human tumour xenografts, and AZD6738 treatments started when the tumours had reached a volume of $0.2-0.3 \mathrm{~cm}^{3}$ (Checkley et al. 2015). Here, we regard treatment responses in terms of two dynamic variables: population cell count or tumour size and percentage of DNA-damaged (i.e. $\gamma \mathrm{H} 2 \mathrm{AX}$-positive) cells. The in vitro and in vivo data used in our current study are available in Supplementary Material S1.

\subsection{The ABM Lattice}

In the model, one agent corresponds to one cancer cell (in the monolayer simulation) or, due to computational costs, one group of cancer cells (in the spheroid simulation). The behaviour and fate of each agent are governed by a set of rules that incorporate both intracellular and environmental dynamic variables using multiscale modelling techniques (Rejniak and Anderson 2011). At the start of an in silico experiment, one initial agent is placed in the centre of the lattice. This initial agent produces daughter agents and ultimately gives rise to a heterogeneous population of agents. When the population has reached an appropriate size (chosen to match the in vitro and in vivo data in the monolayer and spheroid simulations, respectively), AZD6738 anti-cancer treatments commence. The ABM lattice is a square lattice, and every lattice point is either empty or occupied by one agent. If a lattice point is empty, it consists of extracellular solution providing nutrients to cells. In the monolayer simulations, the dispersion of any molecules across the lattice is modelled as instantaneous, and thus the extracellular solution is considered to render the entire lattice homogeneous in terms drug and oxygen concentrations at all times. In the spheroid simulations, however, drug and oxygen molecules are modelled as diffusing over the spheroid and the extracellular environment, and consequently the spheroid lattice will be heterogeneous in terms of drug and oxygen concentrations. Oxygen and drug distribution across the lattice are further discussed in Sects. 2.4 and 2.5, respectively. Since Checkley et al. (2015) report monolayer cell population sizes in units of number of cells, and in vivo tumour sizes in $\mathrm{cm}^{3}$, we here choose to measure simulated monolayer and spheroid sizes using cell counts and volumes, respectively. The ABM lattices are chosen accordingly, as described below.

Monolayer lattice: Cell populations evolve on a two-dimensional square lattice with $100 \times 100$ lattice points, where the spacing in both spatial directions, $x_{1}$ and $x_{2}$, corresponds to one cell diameter.

Spheroid lattice: We simulate (only) a central cross section of the spheroid as an, approximately circular, disc of cells living on a two-dimensional square lattice. This lattice is specifically an $\tilde{L} \times \tilde{L}=1000 \times 1000$ square lattice, with a spacing in both spatial directions $\tilde{x}_{1}$ and $\tilde{x}_{2}$ equal to $40 \mu \mathrm{m}$. The dimensions are chosen in order to allow our 
ABM to simulate the required physical dimensions, whilst keeping computational costs low. Post-simulation time, the two-dimensional cross section of cells is extrapolated to represent a three-dimensional spheroid. This disc-to-spheroid extrapolation process is outlined in Supplementary Material S4.

\subsection{Cell Cycle Model}

In order to capture the influence of ATR and the ATR inhibitor AZD6738 on the cell cycle, we use a probabilistic, rule-based cell cycle model adapted from previous mathematical (non-agent-based) work by Checkley et al. (2015). As illustrated in Fig. 2, this cell cycle model can be represented as a graph with nodes (cell cycle phases or states) that are connected via various paths (phase/state transitions). A cell can be in an undamaged state $(\mathrm{G} 1, \mathrm{~S}$ or $\mathrm{G} 2 / \mathrm{M})$, a replication stress-induced DNA damaged state (D-S) or a dead state, where the cause of cell death is unrepaired replication stress. As ATR is active in the checkpoint in the intra-S phase of the cell cycle, both under undamaged circumstances and in response to DNA damage (Carrassa and Damia 2017), ATR inhibition will inhibit the cell from progressing to the G2/M state in the mathematical cell cycle model. A cell can take different paths through the cell cycle graph, and every time that paths fork, random number generation determines which path will be taken. Every cell commences its life in the G1 state, but thereafter a cell can enter either the $S$ state or the damaged S (D-S) state. The probability that a cell enters the D-S state is denoted $\Pi_{D-S}$ and is calibrated by in vitro data (Checkley et al. 2015). If a cell enters the D-S state, it has a chance to repair itself and enter the $S$ state. If there is no drug in the system, this repair is always achieved, however the repair path is inhibited by the presence of the drug AZD6738. The higher the drug-concentration, the more unlikely it is that a cell in the D-S state will successfully repair itself to the $\mathrm{S}$ state. If a cell in the D-S state fails to repair, it is sentenced to die. Whether a cell in state D-S repairs or dies is decided by comparing a random number, generated from a uniform distribution, to the cell's survival probability, which is influenced by the local drug concentration $C(\bar{x}, t)$, as described in detail in Sect. 2.6. A cell that has successfully reached the $\mathrm{S}$ state continues to the G2/M state, after which it duplicates and starts over in the G1 state again, ready to perform another cell cycle.

In order to allow for asynchronous populations, each agent $i$ on the lattice is assigned an individual doubling time $\tau_{i}$, where $\tau_{i}$ is a random number generated from a normal distribution with mean value $\mu$ and standard deviation $\sigma$. Each agent is attributed an individual cell cycle clock, that determines when the agent should progress to a subsequent state in the cell cycle model. Progression to a subsequent cell cycle state occurs once an agent has spent a certain fraction of its doubling time in its current state. The fraction of the doubling time spent in the G1, S (including D-S) and G2/M states are, respectively, denoted $\Theta_{G 1}, \Theta_{S}$ and $\Theta_{G 2 / M}$, where these values are approximate and chosen from the literature to match values for typical human cells with a rapid doubling time of 24 hours so that $\Theta_{G 1}=11 / 24, \Theta_{S}=8 / 24$ and $\Theta_{G 2 / M}=5 / 24$ (Cooper and Hausman 2007). The fraction of an agent's doubling-time spent in the D-S state, $\Theta_{D-S}$, is on the other hand fitted by in vitro data produced by Checkley 


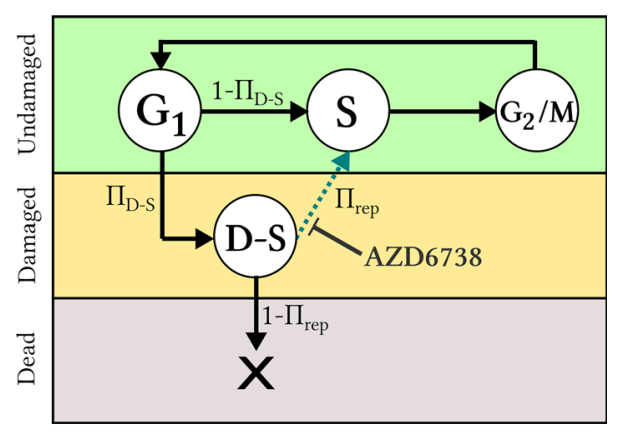

Fig. 2 Cell cycle model: An agent, i.e. a cell (in the monolayer simulation) or a group of cells (in the spheroid simulation), progresses through various states of the cell cycle, where the states correspond to cell cycle phases and are shown as nodes in the graph. Viable (undamaged or damaged) states are shown in circles, whilst the dead state is shown as a cross. Paths illustrate transitions between states, and symbols next to the paths denote the probabilities that the corresponding paths will be taken. The dashed path can be inhibited by an ATR-inhibiting drug, such as AZD6738 (Colour figure online)

et al. (2015), as outlined in Supplementary Material S2. Monolayer and spheroid cell cycle modelling rules are described below.

Monolayer cell cycle model rules: One agent corresponds to one cancer cell that is assigned an individual doubling time $\tau_{i}$. The cell cycle path taken by cell $i$ is governed by drug concentrations and random number generations specific to that cell.

Spheroid cell cycle model rules: One agent comprises a group of identical cancer cells. Each agent is assigned an individual doubling time, $\tau_{i}$, and thus all cells belonging to agent $i$ progress simultaneously and uniformly through the cell cycle model. Random number generations specific to agent $i$ determine which path the agent takes through the cell cycle.

\subsection{Cell Proliferation}

When an agent has completed the mitosis state in the cell cycle model a daughter agent is produced. Each daughter agent is placed on a random lattice point in the (approximately circular) neighbourhood of its parental agent. To accomplish circularlike growth, the model stochastically alternates between placing daughter agents on Moore and von Neumann neighbourhoods of parental agents, as pictorially described in Supplementary Material S3. A daughter agent is allowed to be placed on, up to, a $v$ th-order neighbourhood of its parental agent, but lower-order neighbourhoods (i.e. neighbourhoods closer to the parent) are prioritised and populated first. Modelling rules concerning monolayer and spheroid cell proliferation are outlined below.

Monolayer proliferation rules: In the experimental monolayer in vitro setup, there is no spatial constraint or nutrient deficiency that is inhibiting cell division within the time-course of the experiment. Consequently cells are allowed to divide freely in the monolayer model and we set $v$ to be equal to infinity (with the restriction that agents cannot be placed outside the lattice in the in silico implementation). Although this non-local placement of daughter cells neglects physics, we are not considering spatial 
heterogeneity in the monolayer simulation and therefore cell location does not affect the evaluated simulation results.

Spheroid proliferation rules: In vivo tumours and in vitro spheroids typically consist of a core with non-proliferating cells and a shell of proliferating cells. To accommodate for this, a daughter agent (representing a group of daughter cells) is allowed to be placed on up to a third-order (approximately circular) neighbourhood of its parental agent, so that $\tilde{v}=3$, in accordance with previous mathematical models (Powathil et al. $2012 b$ ). For the spheroid simulation regarded in our current study, $\tilde{v}=3$ matches the experimental in vivo data. However, for other experiments, the value of $\tilde{v}$ should be adjusted to fit the specific cell-line and modelling scenario at hand. When an agent is in the G1 phase of the cell cycle, it scans its environment to see if it has enough resources, in terms of space and nutrients, to commence the process of producing a daughter cell. If not, the cell enters the quiescent phase (Alarcon et al. 2004). Thus in the model, when an agent is in the G1 phase, it continues to progress through the cell cycle model, provided that some free space is available on the lattice within its $\tilde{v}$ th neighbourhood. If this is not the case, the agent exits the cell cycle to enter a quiescent state G0. Should neighbourhood space be made available again, here as a result of anti-cancer targeting, quiescent agents may re-enter the cell cycle.

\subsection{Oxygen Distribution and Influence on Cells}

Tumour growth and treatment responses are highly influenced by intratumoural oxygen levels (Hu et al. 2010; Liapis et al. 2015; Peeters et al. 2015) and severely hypoxic (cancer) cells may proliferate slower than well-oxygenated cells (Alarcon et al. 2004). Monolayer oxygen distribution and responses: In the mathematical monolayer model, all cells are assumed to be well-oxygenated in accordance with the experimental in vitro setup performed by Checkley et al. (2015). Consequently, neither oxygen dynamics nor cellular responses to oxygen levels are incorporated in the monolayer model.

Spheroid oxygen distribution and responses: Within solid tumours, oxygen concentrations typically vary and hypoxic regions are common tumour features (Peeters et al. 2015; Hamis et al. 2020a; Sun et al. 2012). Oxygen gradients are also observed in in vitro tumour spheroids (Voissiere et al. 2017), thus oxygen dynamics across the lattice are here described using a mechanistic diffusion equation, where the oxygen concentration in location $\bar{x}$ at time $t$ is denoted by $K(\bar{x}, t)$ where

$$
\frac{\partial K(\bar{x}, t)}{\partial t}=\nabla \cdot\left(D_{K}(\bar{x}, t) \nabla K(\bar{x}, t)\right)+r_{K} m(\bar{x}, t)-\phi_{K} K(\bar{x}, t) \operatorname{cell}(\bar{x}, t) .
$$

The first term in Eq. 1 describes oxygen diffusion across the lattice, the second term is an oxygen supply term and the third term describes oxygen uptake by cells. Accordingly, $D_{K}(\bar{x}, t)$ is the oxygen diffusion coefficient, and $r_{K}$ and $\phi_{K}$ are supply and consumption coefficients, respectively. The diffusion coefficient for oxygen is known from the literature to be $2.5 \times 10^{-5} \mathrm{~cm}^{2} \mathrm{~s}^{-1}$ (Powathil et al. 2012b). Assuming that oxygen diffuses slower inside the spheroid than outside the spheroid, the oxygen diffusion coefficient is divided by a factor 1.5 if there is a cell in location $\bar{x}$ at time $t$ (Powathil et al. 2012b). The binary factor $m(\bar{x}, t)$ is 1 if the regarded location $\bar{x}$ is 
outside the spheroid boundary at time $t$ and 0 otherwise, i.e. $m(\bar{x}, t)$ is 1 if the regarded lattice point is not occupied by an agent nor completely surrounded by agents, thus oxygen is here modelled as supplied from 'outside the boundary of the spheroid'. Similarly, the binary factor cell $(\bar{x}, t)$ is 1 if there is a viable (non-dead) cell in location $\bar{x}$ at time $t$, and 0 otherwise (Powathil et al. 2012b). Equation 1 is coupled with no-flux boundary conditions, thus the total amount of oxygen in the system will fluctuate over time (Powathil et al. 2012a). A scaled oxygen variable $\hat{K}(\bar{x}, t)$ is introduced in order to express oxygenation levels in percentages (\%) between $0 \%$ and $100 \%$. This scaled oxygen value is computed at every unique time step $t_{u}$ by

$$
\hat{K}\left(\bar{x}, t_{u}\right)=\frac{K\left(\bar{x}, t_{u}\right)}{\max _{\bar{x}, t_{u}} K\left(\bar{x}, t_{u}\right)} \cdot h,
$$

where $\max _{\bar{x}, t_{u}} K\left(\bar{x}, t_{u}\right)$ denotes the maximum occurring $K\left(\bar{x}, t_{u}\right)$-value at the time point $t_{u}$ and $h$ is a scaling factor that is included in order to classify cells with oxygen levels of $10 \%$, or less, as hypoxic (Powathil et al. 2012b; Hamis et al. 2020a). Low cellular oxygen levels have been shown to delay cell cycle progression by inducing arrest in, particularly, the G1 phase of the cell cycle (Alarcon et al. 2004). Consequently, in our model, hypoxic cells display arrest (i.e. delay) in the G1 phase of the cell cycle. In mechanistic Tyson-Novak type cell cycle models (Tyson and Novak 2001; Novak and Tyson 2003, 2004), the cell cycle is governed by a system of ordinary differential equations (ODEs) in which the G1 phase can be inherently elongated under hypoxic conditions by incorporating hypoxia-induced factors into the ODEs (Powathil et al. 2012 b). In the mathematical model discussed in this paper, however, we use agentattributed clocks to model cell cycle progression and thus, in order to achieve a longer G1-phase under hypoxic conditions, we introduce a G1 delay factor (G1DF) (Hamis et al. 2020a) where

$$
G 1 D F(\hat{K}(x, t))= \begin{cases}2 & \text { if } 0 \% \leq \hat{K}(x, t)<1 \% \\ a_{1}+\frac{a_{2}}{a_{3}+\hat{K}(\bar{x}, t)} & \text { if } 1 \% \leq \hat{K}(x, t) \leq 10.5 \% \\ 1 & \text { otherwise }\end{cases}
$$

The G1DF is an approximation for how much the G1 phase is expanded in time as a function of cellular oxygen concentration. It is matched to fit data points extracted from a previous mathematical study by Alarcon et al. (2004), in which a Tyson-Novak cell cycle model is extended to incorporate the action of $\mathrm{p} 27$, a protein that is up-regulated under hypoxia and delays cell cycle progression. Data-fitting yields the parameter values $a_{1}=0.9209, a_{2}=0.8200, a_{3}=-0.2389$ (Hamis et al. 2020a). Thus the fraction of an agent's doubling time spent in the G1 state is $G 1 D F(\hat{K}(\bar{x}, t)) \cdot \Theta_{G 1}$, where $G 1 D F(\hat{K}(\bar{x}, t))=1$ for normoxic cells.

\subsection{Drug Distribution Across the Lattice}

Drug distribution significantly varies between monolayer and spheroid settings. In the regarded monolayer setup, the drug concentration can be regarded as homogeneous, 
whilst heterogeneous drug concentrations must be accounted for when simulating drug distribution across the spheroid. Drug uptake and receptor dynamics is omitted in the model, the drug response of an agent is instead influenced by the drug concentration in the lattice point that it occupies.

Monolayer drug distribution: In the in vitro experiments performed by Checkley et al. (2015), plated cell populations of roughly 1000 cells were treated with AZD6738 in the solvent dimethyl sulphoxide (DMSO). In the mathematical model, we approximate the drug distribution across the lattice to be instantaneous (occurring at treatment time $T_{0}$ ) and homogeneous. We furthermore assume that the drug has a half-life time that exceeds the time course of the experiment, and note that there is no other drug elimination from the in vitro system. In our mathematical model, this is equivalent to there being no drug decay or elimination, hence the drug concentration $C(\bar{x}, t)$, in location $\bar{x}$ at time $t$ is simply given by

$$
C(\bar{x}, t)= \begin{cases}0 \text { everywhere } & \text { if } t<T_{0} \\ \mathbb{C} \text { everywhere } & \text { if } t \geq T_{0}\end{cases}
$$

where $\mathbb{C}$ denotes the applied drug concentration (in units of molarity).

Spheroid drug distribution: The spheroid scenarios simulated in this study are compared to the in vivo experiments performed by Checkley et al. (2015), in which the drug AZD6738, or vehicle in the control case, were administered via oral gavage once per day to female Swiss nude mice. Therefore we include dynamic drug delivery and drug decay in our spheroid simulations. In the mathematical spheroid model, we consider the drug to diffuse through the spheroid from its surrounding, creating a drug gradient within the spheroid. This drug dynamics is modelled using a partial differential equation (PDE), where the concentration of AZD6738 at location $\bar{x}$ at time $t$ is denoted by $C(\bar{x}, t)$ such that

$$
\frac{\partial C(\bar{x}, t)}{\partial t}=\nabla \cdot\left(D_{A Z D}(\bar{x}, t) \nabla C(\bar{x}, t)\right)+p(\bar{x}, t)-\eta_{A Z D} C(\bar{x}, t),
$$

where $D_{A Z D}$ is the diffusion coefficient of the drug AZD6738, and the supply coefficient $p(\bar{x}, t)$ is greater than zero at drug administration times only for lattice points outside the tumour. Assuming first-order kinetics for drug elimination, the drug decay constant $\eta_{A Z D}$ is matched to the reported half-life time of 6 hours for AZD6738 in vivo (Vendetti et al. 2015). Note that the drug decay term here represents all drug elimination from the system, both metabolic and that caused by excretion.

The diffusion rate of a drug is predominantly affected by the molecular size of the drug. More specifically, the diffusion coefficient of a drug is inversely proportional to the square root of the molecular weight of the drug, so that large molecules diffuse more slowly than do small molecules (Dale and Rang 2007). Using this assumption, the drug diffusion coefficient $D_{A Z D}$ is set in relation to the oxygen diffusion coefficient $D_{0_{2}}$, as done in previous mathematical studies (Powathil et al. 2012b). Thus the relationship between the diffusion coefficients corresponds to the square of the inverse relationship 
between the molecular weights, such that

$$
\frac{D_{A Z D}}{D_{0_{2}}}=\frac{\sqrt{\text { molecular weight }\left(O_{2}\right)}}{\sqrt{\text { molecular weight AZD })}}=\sqrt{\frac{31.998 \mathrm{~g} / \mathrm{mol}}{412.512 \mathrm{~g} / \mathrm{mol}}} \approx 0.27851
$$

where the molecular weights are collected from the PubChem database (Kim et al. 2019). Details regarding pharmacokinetics are outside the scope of this study, bioavailability is instead calibrated using extreme case drug scenarios, as described in Supplementary Material S2.

\subsection{Drug Responses}

AZD6738 inhibits DNA repair from the D-S state to the S state in the cell cycle model, as illustrated in Fig. 2, and, in our model, maximal drug effect corresponds to complete repair inhibition. The drug effect is modelled using an agent-based adaptation of the sigmoid Emax model (Holford 2017), a note on the choice of drug model is included in Supplementary Material S6. In the ABM-adapted sigmoidal Emax model used here, the drug effect on a cell in position $\bar{x}$ at time $t$ is given by

$$
E(\bar{x}, t)=E_{\max } \frac{C(\bar{x}, t)^{\gamma}}{E C_{50}^{\gamma}+C(\bar{x}, t)^{\gamma}},
$$

where the drug concentration in lattice point $\bar{x}$ at time $t$ is given by $C(\bar{x}, t)$. $E_{\max }$ denotes the maximal drug effect, here corresponding to complete repair inhibition $\left(E_{\max }=1\right), E C_{50}$ denotes the drug concentration required to achieve half of the maximal drug effect $\left(0.5 \cdot E_{\max }\right)$ and $\gamma$ is the Hill-exponent (Holford 2017). $E C_{50}$ and $\gamma$ are fitted from the in vitro data, as outlined in Supplementary Material S2. When an agent is scheduled to progress from the D-S state in the cell cycle, it has a probability $\Pi_{\text {rep }} \in[0,1]$ to repair, where $\Pi_{\text {rep }}$ is determined by the local drug concentration so that

$$
\Pi_{r e p}(\bar{x}, t)=1-E(\bar{x}, t)
$$

Note that in the absence of drugs, the repair probability is 1 . When a cell dies, it is transformed into a membrane-enclosed 'cell-corpse' (Dale and Rang 2007). In an in vivo setting, this cellular debris is digested by macrophages but in an in vitro setting such 'cell-corpses' may linger on the lattice during the course of the experiment. Post the lethal event (i.e. the D-S to $S$ repair failure) a cell is declared 'dead' in the model after a time $T_{L \rightarrow D}$ has passed (where $L$ stands for 'lethal event' and $D$ stands for 'death'). The parameter $T_{L \rightarrow D}$ is calibrated by in vitro experiments. The differences between modelling rules for monolayer and spheroid drug responses are described below.

Monolayer drug responses: After failure to repair from the D-S state, a cell (i.e. an agent) is considered to be dead after a time $T_{L \rightarrow D}$ has passed. However, a dead cell is never physically removed from the lattice. 
Table 1 In vitro-calibrated parameters

\begin{tabular}{lll}
\hline Section & Parameter & Calibrated Value \\
\hline 2.2 & $\mu, \sigma$ & $24 \mathrm{~h}, 0.5 \mathrm{~h}$ \\
& $\Pi_{D-S}, \theta_{D-S}$ & $0.75,0.03$ \\
2.6 & $\mathrm{EC}_{50}, \gamma$ & $1 \mu \mathrm{M}, 2$ \\
& $T_{L \rightarrow D}$ & $\tau_{i}$ \\
\hline
\end{tabular}

$\mu$ and $\sigma$, respectively, denote the mean value and the standard deviation of the normal distribution from which an agent's cell cycle length is picked. $\Pi_{D-S}$ and $\theta_{D-S}$ denote the probability that an agent enters the D-S state, and the fraction of its cell length spent in the D-S state. $\mathrm{EC}_{50}$ and $\gamma$ denote the half maximal drug concentration and the Hillexponent in the drug response equation (5). $T_{L \rightarrow D}$ denotes the time it takes for an agent to die post-DNA damage repair failure, and $\tau_{i}$ denotes the cell cycle length of agent $i$

Spheroid drug responses: In order to simulate in vivo-like removal of dead cancer cells, an agent (i.e. a group of cells) is declared to be dead and is removed from the lattice after an amount of time $T_{L \rightarrow D}$ post the lethal event (failure to repair).

\subsection{Parameters}

The parameters used in the mathematical model are calibrated by monolayer in vitro data, this calibration process is described in Supplementary Material S2. In the context of quantitative pharmacology, knowledge about a model's robustness is crucial (Visser et al. 2014), therefore we have provided results from the uncertainty and sensitivity analysis in Supplementary Material S9. We performed three different uncertainty and sensitivity analyses techniques, suitable for stochastic agent-based models, namely (i) consistency analysis, (ii) robustness analysis and (iii) Latin hypercube analysis (Hamis et al. 2020b; Alden et al. 2013). Detailed descriptions on how to perform and interpret these techniques are available in an introductory uncertainty and sensitivity analyses review (Hamis et al. 2020b). In accordance with the performed consistency analysis, we run 100 simulations per in silico experiment in order to formulate results (in terms of mean values and standard deviations) that mitigate uncertainty originating from intrinsic model stochasticity.

\subsection{Differences Between Monolayer and Spheroid Modelling Rules}

Modelling rules are adjusted when moving between monolayer and spheroid simulations. Differences between monolayer and spheroid rules are pictorially summarised in Fig. 3. A note on the simplifying modelling assumptions that we have used in this study is provided in Supplementary Material S5.

\subsection{Implementation}

The mathematical model is implemented in an in-house C++ (Stroustrup 1995) framework, in which PDEs are solved using explicit finite difference methods. Simulation 


\begin{tabular}{|c|c|c|}
\hline & monolayer rules & spheroid rules \\
\hline 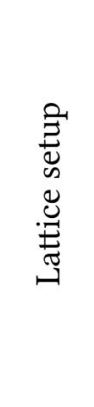 & $\begin{array}{l}\text { A monolayer of cells } \\
\text { is simulated. }\end{array}$ & One agent represents a group of cancer cells. $\begin{array}{r}\begin{array}{r}\text { A center tumour cross- } \\
\text { section is simulated, } \\
\text { from which a tum- } \\
\text { our spheroid is } \\
\text { extrapolated } \\
\text { post sim- } \\
\text { ulation } \\
\text { time. }\end{array} \\
\text { time }\end{array}$ \\
\hline 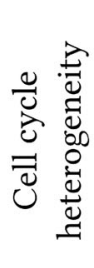 & All cells are cycling. & $\begin{array}{l}\begin{array}{l}\text { Agents that are surrounded by other agents in } v \\
\text { or more orders of their circular neighbourhoods } \\
\text { are quiescent. }\end{array} \\
\text { G1 S OD-S G2/M OG0 }\end{array}$ \\
\hline 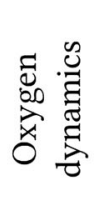 & $\begin{array}{l}\text { All cells are well oxygenated, oxygenation } \\
\text { levels are constant in time and space on the } \\
\text { lattice. }\end{array}$ & $\begin{array}{l}\text { Oxygen diffuses in from outside the } \\
\text { tumour. Hypoxia induces G1 arrest. }\end{array}$ \\
\hline 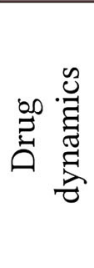 & $\begin{array}{l}\text { Drug concentration } \\
\text { Post drug administration time, drug concen- } \\
\text { tration is constant in time and space on the } \\
\text { lattice. }\end{array}$ & $\begin{array}{l}\text { Drugs diffuse in from outside the tumour, } \\
\text { and drugs are eliminated from the system. }\end{array}$ \\
\hline 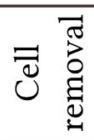 & Dead agents are not removed from the lattice. & $\begin{array}{l}\text { Dead agents are removed from the lattice after a } \\
\text { time } T_{L \rightarrow D} \text {. }\end{array}$ \\
\hline
\end{tabular}

Fig. 3 A summary of the differences between the monolayer and spheroid modelling rules used in the mathematical framework (Colour figure online)

cell-maps are visualised using ParaView (Utkarsh 2015) and data analysis, as well as uncertainty and sensitivity analyses, are performed in MATLAB (MATLAB 2019).

The mathematical framework is first calibrated by in vitro monolayer data produced by Checkley et al. (2015). Thereafter, spheroids subjected to dynamic drug delivery and the removal of dead cells are simulated. Spheroid simulations are then compared to in vivo treatment responses in human tumour xenografts. Two model outputs are considered in the in silico simulations: the fraction of DNA damaged cells in the system and the size of the cancer cell population or tumour spheroid over time. Note 
that, in the model, a cell is classified as DNA-damaged if it is in the D-S state of the cell cycle depicted in Fig. 2. In the experimental setup, DNA damaged cells are labelled as $\gamma$-H2AX positive (Checkley et al. 2015).

\section{Results}

\subsection{Simulating Monolayer Experiments}

In the in vitro experiments, populations of LoVo (human colon carcinoma) cells were exposed to the ATR-inhibiting drug AZD6738 (Checkley et al. 2015). Figure 4 shows monolayer simulation results, specifically the percentage of DNA damaged ( $\gamma$ H2AX-positive) cells over time (left) and the total cell count over time (right). In the simulations, AZD6738 drugs are given at 0 hours, when the cell population has reached a size of approximately 1000 cells. Simulated response curves for six different drug concentrations, including the zero-drug concentration control case, are shown. Also shown in Fig. 4 are in vitro data and results from the mathematical compartmentODE model presented by Checkley et al. (2015) describing the same experimental scenario. Using a minimal-parameter modelling approach, the mathematical framework is calibrated to fit in vitro data points without introducing any variable model parameters. This calibration process is described in Supplementary Material S2.

Our results demonstrate that, post-in vitro monolayer parameterisation, our mathematical framework is able to capture the qualitative nature of in vitro monolayer LoVo cell population growth and drug (AZD6738) responses. The model qualitatively reproduces the asymptotic fraction of DNA damaged cells in the system but fails to match early data points (Fig. 4, left). The sensitivity analysis (Supplementary Material S9) demonstrates that the treatment timing (in relation to the overall cell cycle phase
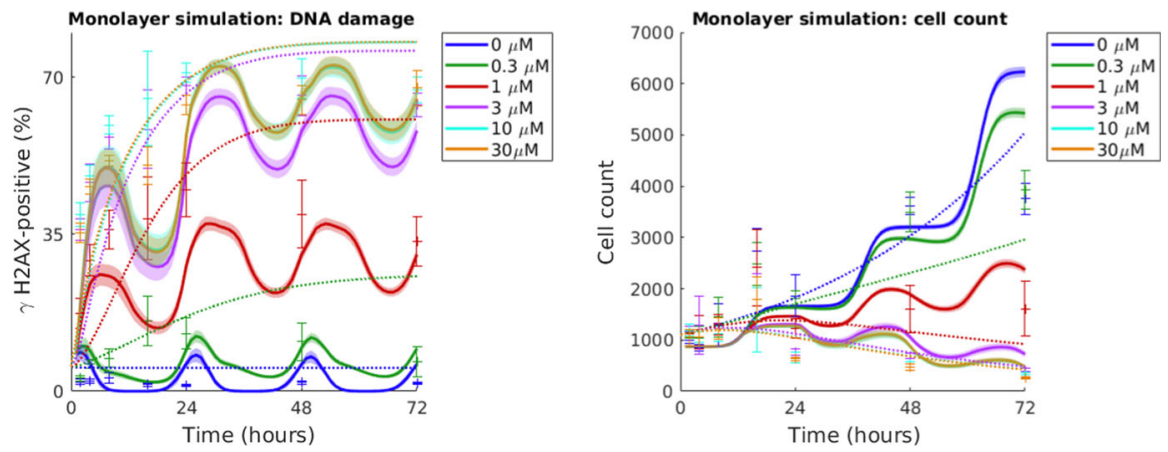

Fig. 4 Simulated monolayer drug response curves are compared to in vitro data and mathematical results from a previously published compartment-ODE model by Checkley et al. (2015). LoVo cells are exposed to drug (AZD6738) at 0 hours. Left: The percentage of $\gamma \mathrm{H} 2 \mathrm{AX}$-positive (DNA-damaged) cells in the system over time. Right: Cell count over time. ABM simulated mean values and standard deviations for 100 in silico runs are shown with solid lines and shaded ribbons, respectively. In vitro data in form of mean values and standard deviations are demonstrated with centre points and error bars (Checkley et al. 2015). Compartment-ODE model results are represented by dotted lines (Colour figure online) 


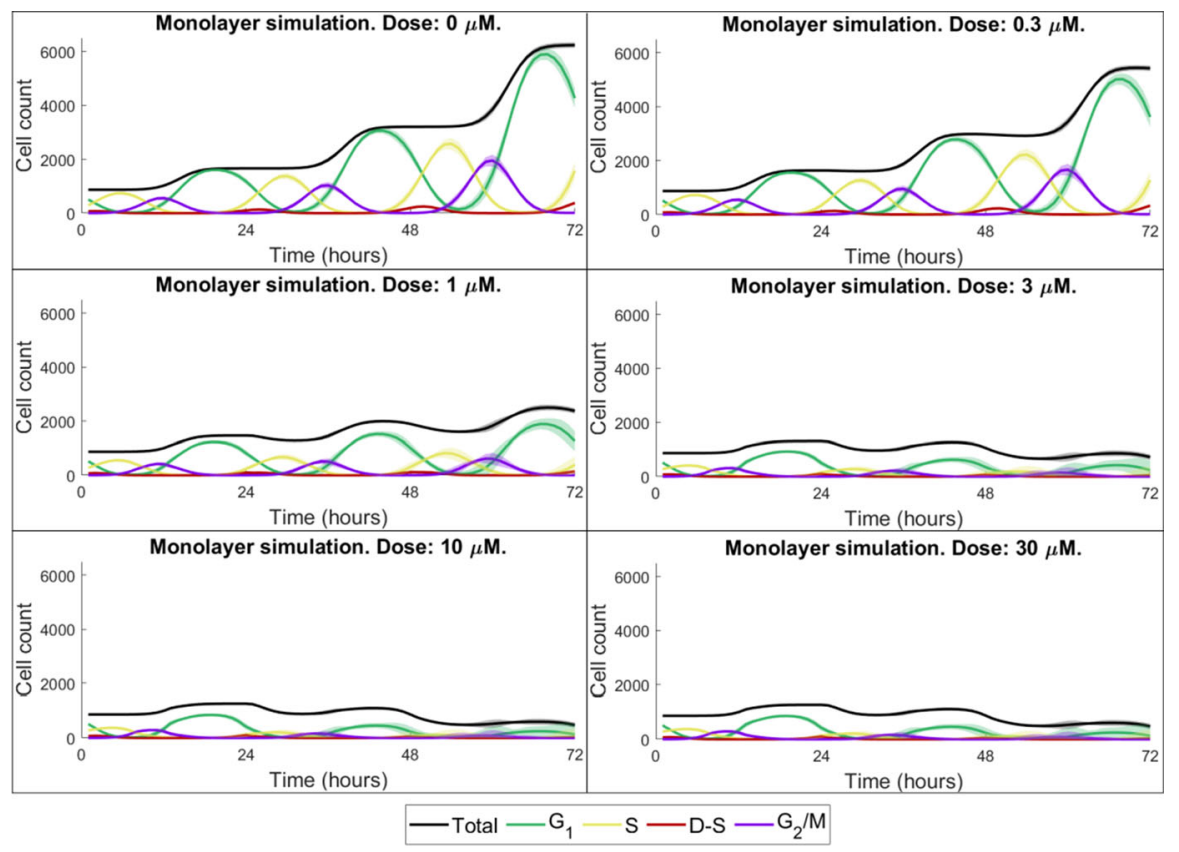

Fig. 5 Cell cycle state-specific monolayer cell counts. Each subplot shows the number of cells in the $\mathrm{G}_{1}$, $\mathrm{S}, \mathrm{D}-\mathrm{S}, \mathrm{G}_{2} / \mathrm{M}$ state, as well as the total cell count, for a specific drug dose. Mean values and standard deviations for 100 in silico runs are shown with solid lines and shaded ribbons, respectively (Colour figure online)

composition of the cancer cell population) notably influences treatment responses in terms of percentage of $\gamma \mathrm{H} 2 \mathrm{AX}$-positive cells. The model parameter calibration process selects for a strong cell cycle synchronisation amongst cancer cells, determined by the model parameter $\sigma$ (Table 1). This strong synchronisation gives rise to oscillatory cell cycle state compositions, as can be seen in Fig. 5 where cell cycle-specific cell counts are plotted over time in response to different drug doses. This strong synchronisation also yields the step-wise growth curves seen in Fig. 4 (right). The experimental error bars in this figure and the numerical cell count data available in Table S1 (Supplementary Material S1) demonstrate that the doubling time of the cell population drastically decreased towards the end of the in vitro experiment and, consequently, our agent-based model was not able to replicate cell count data at 72 hours as the modelling rules and parameters are not updated over time.

The ABM model and Checkley et al.'s (2015) compartment-ODE model are also compared to each other and in vitro data in residual plots available in Supplementary Material S7. In an effort to quantify how well the two mathematical models match the data, the Root Mean Square Errors (RMSEs) are computed between $N$ simulation mean values and data mean values so that $\mathrm{RMSE}=\left(\sum_{i=1}^{N}\left(\operatorname{sim}_{i}-\operatorname{data}_{i}\right)^{2} / N\right)^{1 / 2}$. When comparing DNA damage simulation results to data, the ABM model yields an RMSE of 11.6 percent units, whilst the compartment-ODE model yields an RMSE of 14.6 percent units. When comparing cell count simulation results to data, however, the ABM RMSE is 644 cells whilst the compartment-ODE RMSE is 495 cells. 

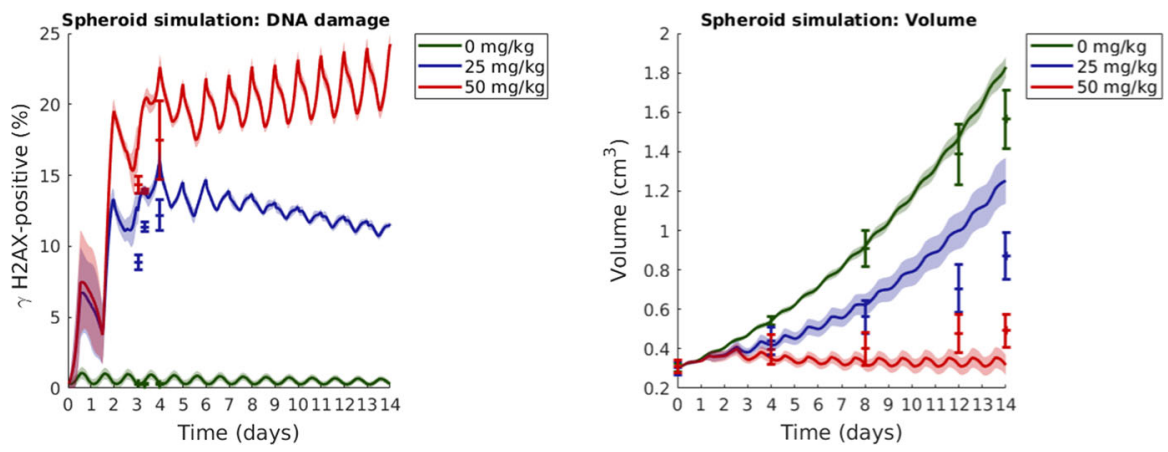

Fig. 6 Simulated spheroid drug response curves are compared to in vivo xenograft data. In both the spheroid simulation and the in vivo experiment, LoVo xenografts are exposed to drug (AZD6738) once daily for 14 days. Left: The percentage of $\gamma \mathrm{H} 2 \mathrm{AX}$-positive (DNA-damaged) cells in the spheroid/xenograft over time. Right: Spheroid/xenograft volume over time. Simulated (spheroid) mean values and standard deviations for 100 in silico runs are shown with solid lines and shaded ribbons, respectively. In vivo data in form of mean values and standard errors are demonstrated with centre points and error bars (Checkley et al. 2015) (Colour figure online)

\subsection{Simulating Spheroid Experiments}

Post-in vitro monolayer calibration, the mathematical framework is used to simulate spheroid experiments, that are compared to the in vivo experiments performed by Checkley et al. (2015) in which LoVo xenografts, that are injected in mice flanks, are treated with AZD6738 once daily for 14 days. The results in Fig. 6 show AZD6738 drug responses in terms of the percentage of DNA damaged $(\gamma \mathrm{H} 2 \mathrm{AX}$-positive $)$ cells (left) and spheroid/tumour volume (right) over time. Simulated response curves to three different drug doses $(0,25$ and $50 \mathrm{mg} / \mathrm{kg})$ and in vivo data are provided in Fig. 6.

Figure 6 (right) demonstrates that our simulated spheroid results qualitatively agree with the in vivo results reported by Checkley et al. (2015) for approximately 12 days post-tumour injection for control case tumours and for approximately 8 days posttumour injection for tumours subjected to drugs. This can be explained by the fact that the behaviour of the agents in our current model does not change over time, when in fact tumours are highly adaptable and responsive to external pressures. It follows that details pertaining to tumour growth and drug sensitivity may vary over time, and in the future work, the agent-based model used in this study can be updated to incorporate variable modelling rules and parameters. Residual plots, comparing ABM simulation results to in vivo data (Checkley et al. 2015) are available in Supplementary Material S7 for DNA damage and spheroid/tumour volume evaluations. The RMSE for DNA results is 3.57 percent units. For the spheroid/tumour volume simulation results, the RMSE is $0.038 \mathrm{~cm}^{3}$ up to and including 8 days, but $0.16 \mathrm{~cm}^{3}$ for the full 14-day simulations.

\section{Discussion}

Simulation results obtained in this study were compared to in vitro and in vivo data and, furthermore, to previous mathematical modelling results produced by Check- 
ley et al. (2015). In their study, Checkley et al. (2015) modelled tumour responses to AZD6738 using coupled ordinary differential equations, where a pharmacokinetic/pharmacodynamic (PK/PD) model of tumour growth was integrated with a mechanistic cell cycle model. Their model is predictive of in vivo xenograft studies and is being used to quantitatively predict dose and scheduling responses in a clinical Phase I trial design (Checkley et al. 2015). Our modelling results qualitatively agree with those produced by Checkley et al. (2015), although two different modelling approaches have been taken: Checkley et al. (2015) regard the tumour as one entity with different compartments whilst we here use a bottom-up modelling approach and regard the tumour as consisting of multiple, distinct agents. Since AZD6738 specifically targets cells that are in the damaged S cell cycle state, we included cell cycle phase resolution into the ABM. Although this modelling approach makes parameter calibration more difficult (compared to more phenomenological models in which the drug acts on all cells), it provides an opportunity to study details about the biological system in silico that are not easily observable in vitro or in vivo. Cell cycle phase details will furthermore be of importance in the future work in which the model will be extended to include combination treatments, as many anti-cancer treatments are cell cycle phase specific (Mills et al. 2018).

Moving drug-response investigations from in vitro to in vivo settings is a key step involved in the process of moving a drug from bench-to-bedside. However, in vivo data are often sparse, as gathering in vivo data is associated with practical, financial and ethical constraints. Plentiful and adaptable in silico data are, on the other hand, easy to produce, and can thus be used as epistemic complements to sparse in vivo data. Well-formulated in silico tools can be extended to investigate various doseschedule scenarios in order to guide in vitro and in vivo experiments. Such in silico experiments may provide a testbed for simulating various mono and combination therapies. In this study we aimed to capture treatment responses in tumour spheroids (with dynamic drug delivery and the removal of dead cells) using monolayer data and modelling rules that are based on chosen 'fundamental' principles that describe how cancer cells in a system behave. Although our spheroid simulations were able to qualitatively mimic the dynamics of in vivo xenografts at early time-points (up to 8 days) post-tumour injection, the model did not match data at later time points. This is to be expected, as the effects of certain biological processes that are present in vivo, but not in vitro (e.g. angiogenesis), do not impact the tumour volume instantly after tumour injection. Thus the spheroid model can, in the future work, be extended to more accurately simulate in vivo scenarios. For example, stromal tumours cells, angiogenesis and metastasis can be included in the model. Moreover, further heterogeneity amongst cancer cells can be incorporated, pertaining to, e.g. drug resistance-related variables. In order to account for mechanical aspects of tumour growth, the approximated cancer cell population/tumour growth model, which allows for daughter agents being placed on non-adjacent lattice point of the parental agent, can be updated to a more realistic proliferation model. Pharmacokinetic details, drug uptake and receptor dynamics can be included in order to make the drug model more detailed. In order to realistically simulate in vivo tumours, effects of the host's immune system can furthermore be incorporated in the model. The observation that anti-cancer drug responses vary between in vitro monolayer, in vitro spheroid and in vivo models has also been 
addressed in a mathematical study by Wallace et al., who used ODE models to simulate neuroblastoma treated with 15 -Deoxy- $P G J_{2}$ in monolayers and spheroids (Wallace et al. 2016). Similar to our modelling approach (Fig. 1), the authors used in vitro data to calibrate a monolayer model, and thereafter extended the model to incorporate spheroid features in order to simulate spheroid dynamics.

The ABM considered in this study is an extension of a mathematical model that has previously been used to study tumour growth and treatment responses to chemotherapy, radiotherapy, hyperthermia and hypoxia-activated prodrugs (Powathil et al. 2012b; Hamis et al. 2020a; Powathil et al. 2015; Hamis et al. 2018, 2019; Bruningk et al. 2018). In recent years, several ABMs have been developed for the purpose of describing various aspects of cancer dynamics (Metzcar et al. 2019), and it should be noted that the modelling approach proposed in Fig. 1 is not conceptually limited to usage with the ABM described in this study. The choice of ABM should be influenced by the research question at hand, the desired level of model details and the available data. Examples of data-driven ABMs are available in a recent review article by Chamseddine and Rejniak (2020) discussing hybrid models, and hybrid modelling techniques, used in the field of mathematical oncology today.

Data-driven modelling, exploitation of existing data and proof-of-concept studies are important steps involved in current and future procedure for enabling mathematical modelling in systems medicine, as argued in a report by Wolkenhauer et al. (2014). A pipeline for predicting therapy outcomes using data-driven mathematical modelling is proposed by Brady and Enderling (2019) in a recent publication. Despite the fact that mathematical modelling is becoming increasingly popular in the pharmaceutical industry, there are not that many ABMs present in the pharmaceutical scene (Cosgrove et al. 2015). We argue that this is a missed opportunity in the context of oncology, as ABMs naturally capture the heterogeneous nature of tumours, which is known to complicate treatments. As multiscale ABMs organically enable the integration of data across various scales in time and space, it follows that they are useful to the interdisciplinary team that wishes to combine data and knowledge from its team members. Following interdisciplinary collaborations between clinicians, biologists and mathematicians, mathematical modelling may be used to enable in silico informed drug development.

Supplementary Information The online version contains supplementary material available at https://doi. org/10.1007/s11538-021-00935-y.

Availability of data and materials All in vitro and in vivo data used in this study are gathered from Checkley et al. (2015) and are listed in Supplementary Material S1.

\section{Declarations}

Conflict of interest The authors declare that they have no conflict of interest.

Code availability (software application or custom code) Project code is available on the code hosting platform GitHub at https://github.com/SJHamis/DDRinhibitors. 
Open Access This article is licensed under a Creative Commons Attribution 4.0 International License, which permits use, sharing, adaptation, distribution and reproduction in any medium or format, as long as you give appropriate credit to the original author(s) and the source, provide a link to the Creative Commons licence, and indicate if changes were made. The images or other third party material in this article are included in the article's Creative Commons licence, unless indicated otherwise in a credit line to the material. If material is not included in the article's Creative Commons licence and your intended use is not permitted by statutory regulation or exceeds the permitted use, you will need to obtain permission directly from the copyright holder. To view a copy of this licence, visit http://creativecommons.org/licenses/by/4.0/.

\section{References}

Alarcon T, Byrne HM, Maini PK (2004) A mathematical model of the effects of hypoxia on the cell-cycle of normal and cancer cells. J Theor Biol 229:395-411

Alden K, Read M, Timmis J, Andrews PS, Veiga-Fernandes H, Coles M (2013) Spartan: a comprehensive tool for understanding uncertainty in simulations of biological systems. PLoS Comput Biol 9:e1002916

Brady R, Enderling H (2019) Mathematical models of cancer: when to predict novel therapies, and when not to. Bull Math Biol 81:3722-3731

Brady-Nicholls R, Nagy JD, Gerke TA, Zhang T, Wang AZ, Zhang J, Gatenby RA, Enderling H (2020) Prostate-specific antigen dynamics predict individual responses to intermittent androgen deprivation. Nat Commun 11:1750

Bruningk S, Powathil G, Ziegenhein P, Ijaz J, Rivens I, Nill S, Chaplain M, Oelfke U, Ter Haar G (2018) Combining radiation with hyperthermia: a multiscale model informed by in vitro experiments. $\mathrm{J} R$ Soc Interface 15(138):20170681

Bruno R, Bottino D, de Alwis DP, Fojo AT, Guedj J, Liu C, Swanson KR, Zheng J, Zheng Y, Jin JY (2020) Progress and opportunities to advance clinical cancer therapeutics using tumor dynamic models. Clin Cancer Res 26:1787-1795

Carrassa L, Damia G (2017) DNA damage response inhibitors: mechanisms and potential applications in cancer therapy. Cancer Treat Rev 60:139-151

Chamseddine IM, Rejniak KA (2020) Hybrid modeling frameworks of tumor development and treatment. Wiley Interdiscip Rev Syst Biol Med 12:e1461

Checkley S, MacCallum L, Yates J, Jasper P, Luo H, Tolsma J, Bendtsen C (2015) Bridging the gap between in vitro and in vivo: dose and schedule predictions for the ATR inhibitor AZD6738. Sci Rep 5:13545

Cooper GM, Hausman RE (2007) The cell: a molecular approach, 4th edn. ASM Press, Washington, DC

Cosgrove J, Butler J, Alden K, Read M, Kumar V, Cucurull-Sanchez L, Timmis J, Coles M (2015) Agentbased modeling in systems pharmacology. CPT Pharmacometrics Syst Pharmacol 4:615-629

Dale MM, Rang HP (2007) Rang \& Dale's pharmacology. Churchill Livingstone, Edinburgh

Foote KM, Nissink JWM, McGuire T, Turner P, Guichard S, Yates JWT, Lau A, Blades K, Heathcote D, Odedra R, Wilkinson G, Wilson Z, Wood CM, Jewsbury PJ (2018) Discovery and characterization of AZD6738, a potent inhibitor of ataxia telangiectasia mutated and Rad3 related (ATR) kinase with application as an anticancer agent. J Med Chem 61:9889-9907

Ghaffarizadeh A, Heiland R, Friedman SH, Mumenthaler SM, Macklin P (2018) PhysiCell: an open source physics-based cell simulator for 3-D multicellular systems. PLoS Comput Biol 14:e1005991

Hamis S, Nithiarasu P, Powathil GG (2018) What does not kill a tumour may make it stronger: in silico insights into chemotherapeutic drug resistance. J Theor Biol 454:253-267

Hamis S, Powathil GG, Chaplain MAJ (2019) Blackboard to bedside: a mathematical modeling bottom-up approach toward personalized cancer treatments. JCO Clin Cancer Inform 3:1-11

Hamis S, Kohandel M, Dubois LJ, Yaromina A, Lambin P, Powathil GG (2020a) Combining hypoxiaactivated prodrugs and radiotherapy in silico: impact of treatment scheduling and the intra-tumoural oxygen landscape. PLoS Comput Biol 16:e1008041

Hamis S, Stratiev S, Powathil GG (2020b) Uncertainty and sensitivity analyses methods for agent-based mathematical models: an introductory review. The Physics of Cancer: Research Advances, ed. Bernard Gerstman. World Scientific Publishing, Singapore

Holford N (2017) Pharmacodynamic principles and the time course of immediate drug effects. Transl Clin Pharmacol 4:157-161 
Hu J, Handisides DR, Van Valckenborgh E, De Raeve H, Menu E, Vande Broek I, Liu Q, Sun JD, Van Camp B, Hart CP, Vanderkerken K (2010) Targeting the multiple myeloma hypoxic niche with TH-302, a hypoxia-activated prodrug. Blood 116:1524-1527

Kim S, Chen J, Cheng T, Gindulyte A, He J, He S, Li Q, Shoemaker BA, Thiessen PA, Yu B, Zaslavsky L, Zhang J, Bolton EE (2019) PubChem 2019 update: improved access to chemical data. Nucleic Acids Res 47:D1102-D1109

Liapis V, Labrinidis A, Zinonos I, Hay S, Ponomarev V, Panagopoulos V, DeNichilo M, Ingman W, Atkins GJ, Findlay DM, Zannettino AC, Evdokiou A (2015) Hypoxia-activated pro-drug TH-302 exhibits potent tumor suppressive activity and cooperates with chemotherapy against osteosarcoma. Cancer Lett 357:160-169

MATLAB (2019) version 1.8.0_202 (R2019n). The MathWorks Inc., Natick

Mei L, Zhang J, He K, Zhang J (2019) Ataxia telangiectasia and Rad3-related inhibitors and cancer therapy: where we stand. J Hematol Oncol 12:43

Metzcar J, Wang Y, Heiland R, Macklin P (2019) A review of cell-based computational modeling in cancer biology. JCO Clin Cancer Inform 3:1-13

Mills CC, Kolb EA, Sampson VB (2018) Development of chemotherapy with cell-cycle inhibitors for adult and pediatric cancer therapy. Cancer Res 78:320-325

Minchom A, Aversa C, Lopez J (2018) Dancing with the DNA damage response: next-generation anti-cancer therapeutic strategies. Ther Adv Med Oncol 10:1758835918786658

Mirams GR, Arthurs CJ, Bernabeu MO, Bordas R, Cooper J, Corrias A, Davit Y, Dunn SJ, Fletcher AG, Harvey DG, Marsh ME, Osborne JM, Pathmanathan P, Pitt-Francis J, Southern J, Zemzemi N, Gavaghan DJ (2013) Chaste: an open source C++ library for computational physiology and biology. PLoS Comput Biol 9:e1002970

Nam AR, Jin MH, Park JE, Bang JH, Oh DY, Bang YJ (2019) Therapeutic targeting of the DNA damage response using an ATR inhibitor in biliary tract cancer. Cancer Res Treat 51(3):1167-1179

Novak B, Tyson JJ (2003) Modelling the controls of the eukaryotic cell cycle. Biochem Soc Trans 31:15261529

Novak B, Tyson JJ (2004) A model for restriction point control of the mammalian cell cycle. J Theor Biol 230:563-579

Nunes AS, Barros AS, Costa EC, Moreira AF, Correia IJ (2019) 3D tumor spheroids as in vitro models to mimic in vivo human solid tumors resistance to therapeutic drugs. Biotechnol Bioeng 116:206-226

Peeters SG, Zegers CM, Biemans R, Lieuwes NG, van Stiphout RG, Yaromina A, Sun JD, Hart CP, Windhorst AD, van Elmpt W, Dubois LJ, Lambin P (2015) TH-302 in combination with radiotherapy enhances the therapeutic outcome and is associated with pretreatment [18F]HX4 hypoxia PET imaging. Clin Cancer Res 21:2984-2992

Powathil G, Kohandel M, Milosevic M, Sivaloganathan S (2012a) Modeling the spatial distribution of chronic tumor hypoxia: implications for experimental and clinical studies. Comput Math Methods Med 2012:410602

Powathil GG, Gordon KE, Hill LA, Chaplain MA (2012b) Modelling the effects of cell-cycle heterogeneity on the response of a solid tumour to chemotherapy: biological insights from a hybrid multiscale cellular automaton model. J Theor Biol 308:1-19

Powathil GG, Swat M, Chaplain MA (2015) Systems oncology: towards patient-specific treatment regimes informed by multiscale mathematical modelling. Semin Cancer Biol 30:13-20

Rejniak KA, Anderson AR (2011) Hybrid models of tumor growth. Wiley Interdiscip Rev Syst Biol Med 3:115-125

Rockne RC, Hawkins-Daarud A, Swanson KR, Sluka JP, Glazier JA, Macklin P, Hormuth DA, Jarrett AM, Lima EABF, Tinsley Oden J, Biros G, Yankeelov TE, Curtius K, Al Bakir I, Wodarz D, Komarova N, Aparicio L, Bordyuh M, Rabadan R, Finley SD, Enderling H, Caudell J, Moros EG, Anderson ARA, Gatenby RA, Kaznatcheev A, Jeavons P, Krishnan N, Pelesko J, Wadhwa RR, Yoon N, Nichol D, Marusyk A, Hinczewski M, Scott JG (2019) The 2019 mathematical oncology roadmap. Phys Biol 16:041005

Scott JG, Maini PK, Anderson ARA, Fletcher AG (2020) Inferring tumour proliferative organisation from phylogenetic tree measures in a computational model. Syst Biol 69(4):623-637

Stephanou A, Fanchon E, Innominato PF, Ballesta A (2018) Systems biology, systems medicine, systems pharmacology: the what and the why. Acta Biotheor 66:345-365

Stroustrup B (1995) The C++ programming language. Addison-Wesley, Boston 
Sun JD, Liu Q, Wang J, Ahluwalia D, Ferraro D, Wang Y, Duan JX, Ammons WS, Curd JG, Matteucci MD, Hart CP (2012) Selective tumor hypoxia targeting by hypoxia-activated prodrug TH-302 inhibits tumor growth in preclinical models of cancer. Clin Cancer Res 18:758-770

Sundar R, Brown J, Ingles Russo A, Yap TA (2017) Targeting ATR in cancer medicine. Curr Probl Cancer 41:302-315

Swat MH, Thomas GL, Belmonte JM, Shirinifard A, Hmeljak D, Glazier JA (2012) Multi-scale modeling of tissues using CompuCell3D. Methods Cell Biol 110:325-366

Tu X, Kahila MM, Zhou Q, Yu J, Kalari KR, Wang L, Harmsen WS, Yuan J, Boughey JC, Goetz MP, Sarkaria JN, Lou Z, Mutter RW (2018) ATR inhibition is a promising radiosensitizing strategy for triple-negative breast cancer. Mol Cancer Ther 17:2462-2472

Tyson JJ, Novak B (2001) Regulation of the eukaryotic cell cycle: molecular antagonism, hysteresis, and irreversible transitions. J Theor Biol 210:249-263

Utkarsh A (2015) The ParaView guide: a parallel visualization application, ISBN: 978-1930934306. Kitware

Vendetti FP, Lau A, Schamus S, Conrads TP, O'Connor MJ, Bakkenist CJ (2015) The orally active and bioavailable ATR kinase inhibitor AZD6738 potentiates the anti-tumor effects of cisplatin to resolve ATM-deficient non-small cell lung cancer in vivo. Oncotarget 6:44289-44305

Visser SA, de Alwis DP, Kerbusch T, Stone JA, Allerheiligen SR (2014) Implementation of quantitative and systems pharmacology in large pharma. CPT Pharmacometrics Syst Pharmacol 3:e142

Voissiere A, Jouberton E, Maubert E, Degoul F, Peyrode C, Chezal JM, Miot-Noirault E (2017) Development and characterization of a human three-dimensional chondrosarcoma culture for in vitro drug testing. PLoS ONE 12:e0181340

Wallace DI, Dunham A, Chen PX, Chen M, Huynh M, Rheingold E, Prosper O (2016) A model for spheroid versus monolayer response of SK-N-SH neuroblastoma cells to treatment with 15-deoxyPGJ2. Comput Math Methods Med 2016:3628124

Wolkenhauer O, Auffray C, Brass O, Clairambault J, Deutsch A, Drasdo D, Gervasio F, Preziosi L, Maini P, Marciniak-Czochra A, Kossow C, Kuepfer L, Rateitschak K, Ramis-Conde I, Ribba B, Schuppert A, Smallwood R, Stamatakos G, Winter F, Byrne H (2014) Enabling multiscale modeling in systems medicine. Genome Med 6:21

Publisher's Note Springer Nature remains neutral with regard to jurisdictional claims in published maps and institutional affiliations. 